\title{
La política como temática de estudio en las revistas hispánicas de comunicación
}

\author{
Politics as a topic of study in Hispanic \\ communication journals
}

Valeriano Piñeiro-Naval* (D)https://orcid.org/0000-0001-9521-3364
University of Beira Interior, Portugal, vale.naval@labcom.ubi.pt

Ricardo Morais (D)https://orcid.org/0000-0001-8827-0299

University of Beira Interior, Portugal, ricardo.morais@labcom.ubi.pt

*Autor para

correspondencia:

Valeriano Piñeiro-Naval

vale.naval@labcom.ubi.pt

Recepción: 17/06/20

Aprobación: 22/07/20

Publicación: 28/09/20

\begin{abstract}
The goal of this paper is to observe the relationship between the areas of communication and politics, particularly exploring the way academic production has been verified by this intersection. Thus, we analyze how the articles published in the main Hispanic communication journals adopt the subject of politics over the fiveyear period 2013-2017. Through a methodological procedure that combines bibliometric analysis with meta-research, we collected data on the objects of study, the theories or the empirical techniques used in the 167 manuscripts comprised in the sample. The main results indicate an increasing trend in the annual number of publications and its consequent impact. From an operational point of view, the authors frequently turn to framing theory and content analysis to address political issues that are increasingly reported on social media.
\end{abstract}

Key words: communication, politics, meta-research, academic articles, Hispanic journals.

Resumen: Este trabajo tiene por objetivo observar las relaciones entre las áreas de la comunicación y la política, explorando, en particular, el modo mediante el cual la producción académica ha reflejado esta intersección. Analizamos cómo los artículos publicados en las principales revistas hispánicas de comunicación abordan la temática de la política durante el quinquenio 2013-2017. A través de un procedimiento metodológico que combina el análisis bibliométrico con la meta-investigación, tomamos datos sobre los objetos de estudio, las teorías o las técnicas empíricas empleadas en los 167 manuscritos que conforman la muestra. Los principales resultados indican una tendencia creciente en el número anual de publicaciones y su consiguiente impacto. Desde el punto de vista operativo, los autores recurren habitualmente a la teoría del framing y al análisis de contenido para abordar los asuntos políticos que, cada vez con más frecuencia, son divulgados a través de las redes sociales.

Palabras clave: comunicación, política, meta-investigación, artículos académicos, revistas hispánicas. 


\section{Introducción $^{1}$}

La comunidad académica ha recuperado, en los últimos tiempos, un gran interés por la relación que existe entre la comunicación y la política (Correia et al., 2010; Restrepo-Echavarría, 2019; Sierra y Rodríguez-Virgili, 2020). Sin la pretensión de ser exhaustivos, algunas de las causas de esta renovada atención residen, en primer lugar, en las múltiples transformaciones que han afectado a las sociedades democráticas contemporáneas y que han incidido, de manera significativa, en las organizaciones políticas, el sistema mediático y el papel de la ciudadanía (Prior et al., 2016). Por otro lado, la mediatización y la "espectacularización" de la vida política (politainment) y el abanico de posibilidades que tanto las tecnologías de la información y la comunicación (en adelante, TIC) como las redes sociales han introducido en el panorama actual (Campos-Domínguez, 2017), han servido de acicates para impulsar multitud de iniciativas científicas.

Cabe aclarar, sin embargo, que el propósito del presente estudio no radica en examinar cada una de estas disciplinas por separado, sino en observar la naturaleza de su intersección. En particular, nos interesa el área situada a medio camino entre la comunicación y la política, cuya dinámica se apoya en determinados preceptos epistemológicos y rutinas metodológicas inherentes también a otras disciplinas, como la sociología política, la lingüística e incluso la psicología social (Prior et al., 2016).

A nivel histórico, la progresiva implantación del sufragio universal y el protagonismo de los medios de comunicación de masas en el proceso político (Correia et al., 2010) fueron los estímulos primordiales que impulsaron el desarrollo inicial de este campo. Más recientemente, el encuentro de estas disciplinas ha originado trabajos acerca de campañas electorales, uso de medios digitales por parte de actores políticos, estudios sobre marketing, periodismo y política, o investigaciones sobre deliberación cívica, participación ciudadana y engagement (Ballesteros y Díez-Garrido, 2018; Muñiz et al., 2016); este último vinculado, de forma muy asidua, a los estudios sobre Web 2.0.

Es en dicho contexto, marcado por la reconfiguración de las relaciones existentes y por la emergencia de nuevas conexiones, donde se ubica nuestro trabajo, en el cual exploramos las múltiples posibilidades de investigación que manan de la confluencia entre comunicación y política.

1 Este trabajo parte de un proyecto financiado por la Fundação para a Ciência e a Tecnologia (Ref./número: SFRH/BPD/122402/2016) de Portugal. 
Desde este planteamiento, Mancinas-Chávez et al. (2016: 242) aseguran que "la divulgación científica de los resultados de investigaciones que se realizan desde todas las ciencias y ámbitos del conocimiento es fundamental para el crecimiento de cada una de las áreas". En este sentido, el nivel de producción académica en torno a un determinado campo epistemológico actúa como un indicador que permite comprender su relevancia y evolución a lo largo de un periodo específico (Pérez-Montoro, 2016). Pues bien, en el presente estudio efectuamos una recogida de artículos divulgados en las principales revistas hispánicas de comunicación durante el quinquenio 2013-2017, con el fin de identificar las líneas conceptuales e instrumentales que caracterizan a estos manuscritos. De forma más específica, se buscó:

- $\mathrm{OE}_{1}$ : describir la producción científica sobre comunicación política en términos bibliométricos.

- $\mathrm{OE}_{2}$ : identificar los elementos nucleares en que se apoya esta producción desde los puntos de vista teórico y metodológico.

- OE3: comparar el perfil de los trabajos en función del origen geográfico de las revistas donde se divulgan; a saber, las cabeceras españolas frente a las hispanoamericanas.

Respecto a la estructura del artículo, llevamos a cabo, en el primer bloque, un breve repaso cronológico donde señalamos los hitos más destacados en la unión entre comunicación y política. A continuación, se abordan las tendencias más recientes, entre las que sobresale el predominio de las TIC y las redes sociales. Cerramos el estado del arte con una discusión acerca de las metodologías más extendidas. La parte central presenta la estrategia adoptada para la selección de la muestra de estudio y su consiguiente análisis, así como los resultados estadísticos obtenidos. Por último, la discusión de los principales hallazgos nos permitirá exponer las conclusiones, así como las limitaciones y líneas futuras de trabajo.

\section{Estado del arte}

\section{Hitos en la intersección entre comunicación y politica}

En opinión de Rogers (2004), los precursores del estudio de la comunicación de masas y la comunicación política compartían un interés común por los efectos de los medios; dándose, a menudo, un trasvase de investigadores de una corriente a otra. Cabe recalcar que ambas tradiciones estuvieron condicionadas, desde su eclosión, por el contexto sociopolítico derivado de la Primera Guerra Mundial (Jamieson y Kenski, 2017). Destacan aquí autores 
como Lippmann (1922 y 1925) y Lasswell (1927), quienes, en las décadas de 1920 y 1930, se centraron en la monitorización de la propaganda y sus consecuencias en la opinión pública.

Tras esta fase inaugural, el temor ante el estallido de una nueva guerra de alcance planetario y el auge de los movimientos ideológicos extremistas (fascismo y comunismo) estimularon una apuesta más decidida por aquellos trabajos que medían las percepciones de la ciudadanía y su posible manipulación (Robinson, 2019). Entre las décadas de 1950 y 1960 se crearía la Rockefeller Foundation, que financiaría un gran conjunto de iniciativas que buscaban comprender los efectos de los medios de comunicación en la sociedad (Karpf et al., 2015). Al amparo de esta financiación, surgen proyectos como la Office of Radio Research de la Universidad de Columbia, sin olvidar el lanzamiento de la revista Public Opinion Quarterly (Robinson, 2019).

Por otra parte, el trabajo liderado por Lazarsfeld et al. (1968), titulado “The People's Choice”, también es particularmente relevante. Mediante un conjunto de encuestas, estos pioneros observaron la incidencia de la comunicación en los electores durante distintas campañas, concluyendo que los efectos de los medios eran más limitados de lo que se pensaba a priori. Estos hallazgos, sumados a las tendencias vinculadas al "behaviorismo" (Rogers, 2004), serían los detonantes, tal y como explican Karpf et al. (2015), de la puesta en marcha de la revista Political Communication, que comenzaba su andadura en 1975 erigiéndose en el primer foro dedicado, específicamente, a la producción académica sobre comunicación política.

En las décadas de 1970 y 1980 este tipo de investigación comienza a nutrirse de otras áreas como la psicología y la ciencia política, alejándose de la vertiente sociológica inicial. Gran parte de los preceptos teóricos que pasaron a definir el campo están reunidos en el libro "Handbook of Political Communication" (Nimmo y Sanders, 1981), entre los que ya comenzaban a destacar la agenda-setting, el framing o el priming; y que relegaron los trabajos de Lippmann (1922 y 1925) o Lasswell (1927) a un segundo plano, así como las teorías y metodologías utilizadas por estos. ${ }^{2}$

El hecho de ceñirse a un conjunto reducido de elementos conceptuales e instrumentales es motivo de advertencia por parte de la comunidad científica reciente (Bennett e Iyengar, 2008; Bimber, 2015; Kparf et al., 2015; Nielsen, 2014; Robinson, 2019), dadas las dificultades de adaptación de la disciplina a los nuevos tiempos. En consecuencia, la cuestión que se plantea es la de

2 Las líneas de investigación de Lippmann o Lasswell aludían a la influencia de la propaganda, el poder de los medios de comunicación y el estudio de la opinión pública, mediante un agregado de metodologías mixtas que cruzaban técnicas cuantitativas y cualitativas. 
saber si -al contrario de lo que advierten algunos expertos- el campo de la comunicación política está preparado para abordar los objetos de análisis del siglo XXI, así como los consiguientes cambios sociales y tecnológicos que estos provocan.

\section{La importancia de Internet y las redes sociales en la comunicación politica}

Al hilo de las perspectivas teóricas y metodológicas privilegiadas desde finales del siglo pasado, y sus notables limitaciones para adaptarse a los entornos digitales, en este segundo epígrafe focalizamos nuestra atención en las innovaciones que los nuevos medios han introducido en la comunicación política. Del mismo modo, otra de las razones que nos impulsa a reparar en la importancia de Internet y, sobre todo, de las redes sociales se debe a que el periodo de análisis considerado en este trabajo (2013-2017) está marcado por el impacto de las TIC en todas las esferas de la sociedad.

A tenor de las distintas bases de datos existentes, entre las que sobresalen Web of Science y Scopus, la producción científica en esta área ha experimentado un incremento considerable. De acuerdo con Rodríguez-Estrada et al. (2017), la producción de 2006 a 2016 presente en Scopus triplica el volumen de trabajos publicados en comparación con la década anterior. Sin embargo, hemos de remontarnos a los años noventa como punto de partida de un nuevo modelo de comunicación política que, en aquel entonces, ya comenzaba a introducir las innovaciones derivadas del empleo de Internet.

Más tarde, como bien señala Rodríguez-Andrés (2016), las dos campañas electorales de Obama (de 2008 y 2012) supondrían casos paradigmáticos de lo que se ha dado en denominar "comunicación política 2.0"; un modelo que ha alterado las dinámicas jerárquicas y verticales que se habían establecido entre las organizaciones políticas, los medios y la ciudadanía. Este cambio tan significativo no pasó inadvertido para los investigadores, que rápidamente comenzaron a prestar atención al modo en que "los políticos están utilizando Internet, redes sociales y, en general, las nuevas tecnologías en el marco de sus estrategias de comunicación y de campaña" (RodríguezAndrés, 2016: 2), ya que su interacción con medios y usuarios es ahora mucho más horizontal e inmediata.

Con el objetivo de observar la relación entre la comunicación política y las redes sociales, Sierra y Rodríguez-Virgili (2020) analizaron los artículos publicados en cuatro de las revistas con mayor impacto del área, como son: Journal of Communication, New Media \& Society, Political Communication e International Journal of Press/Politics. Tras su estudio, estos autores verifica- 
ron un acusado aumento de los artículos centrados en la dimensión política de las redes sociales. Otros hallazgos apreciables aludían al carácter empírico de los manuscritos que, por otra parte, solían recurrir a aquellos paradigmas teóricos dominantes; es decir, agenda setting y framing. Esta circunstancia es congruente con el estudio de Chung et al. (2013), en el que se resaltaba la relevancia de ambos preceptos conceptuales en el campo de la comunicación en general. Asimismo, Piñeiro-Naval y Mangana (2019) también notaban a la política como la temática más frecuente en aquellos artículos que usaban la teoría del framing en las revistas hispánicas de comunicación; concretamente, 26,9\% de los 104 manuscritos examinados durante la década de 2007 a 2016.

Por otro lado, Lotero-Echeverri et al. (2020) subrayan, tras un análisis bibliométrico de 537 artículos indexados en Scopus y publicados por investigadores iberoamericanos, el predominio de los estudios sobre comunicación digital y redes sociales en el campo de la comunicación y la opinión pública. La preeminencia de métodos cuantitativos, como el análisis de contenido $(33,5 \%)$ y la encuesta $(26,4 \%)$, también es un rasgo característico de la muestra estudiada por Lotero-Echeverri et al. (2020). En sintonía con este hallazgo, Sierra y Rodríguez-Virgili (2020) inciden, nuevamente, en la naturaleza cuantitativa de la mayoría de los estudios, lo cual nos permite dar paso al siguiente apartado del trabajo.

\section{¿Nuevas metodologías para el estudio de la comunicación politica?}

Como señalábamos en secciones anteriores, el nuevo ecosistema mediático impulsado por las TIC ha supuesto un gran reto a nivel teórico. En lo que a los procedimientos empíricos concierne, "la cuestión específica del método debe estar a la vanguardia del debate académico sobre el futuro de la investigación en comunicación política" (Kparf et al., 2015: 1890), pues los desafíos a este nivel no son menos exigentes que en el plano conceptual.

Tradicionalmente, este campo de estudio ha incorporado herramientas desarrolladas por la psicología social, ciertos elementos de la ciencia política y otros pertenecientes a la corriente de la comunicación de masas (Nielsen, 2014). Estas razones ayudan a explicar, en parte, una visión positivista centrada en el empleo sistemático de métodos cuantitativos destinados a medir tanto el contenido de los medios como sus efectos en la opinión pública (Robinson, 2019).

Este cariz eminentemente cuantitativo se pone de manifiesto, una vez más, en una meta-investigación llevada a cabo por Graber y Smith (2005), en la cual manejan una muestra de 137 artículos publicados, desde 2000 
hasta 2003, en determinadas cabeceras de referencia, como: American Political Science Review, American Journal of Political Science, Journal of Politics, Communication Research, Journal of Communication y Journalism \& Mass Communication Quarterly. Tras su análisis, concluyen que las campañas electorales $(15,3 \%)$ y los nuevos medios $(10,2 \%)$ son los objetos de estudio más habituales, y que "el análisis de contenido sigue siendo el método más utilizado para examinar mensajes políticos” (Graber y Smith, 2005: 491).

En esta misma línea, Kparf et al. (2015) seleccionaron un total de 258 artículos publicados en Political Communication desde 2003 hasta 2015. Del corpus total, únicamente $16,7 \%$ de los trabajos son cualitativos. Por otro lado, sus postulados son reforzados cuando aluden a una monografía indispensable titulada: "Sourcebook for Political Communication Research" (Bucy y Holbert, 2013), ya que en ella abundan los análisis de contenido, las encuestas y los experimentos, que permiten plantear preguntas muy concretas sobre los fenómenos políticos (Kparf et al., 2015).

Una mayor apuesta por los métodos cualitativos no implicaría, en opinión de estos autores, el abandono de la cuantificación de los contenidos, procesos y efectos, sino una mayor receptividad a la combinación de métodos o "triangulación" (Denzin, 2012). No obstante, Kparf et al. (2015) también sostienen que la implementación de estas iniciativas precisa que los académicos estén tan rigurosamente entrenados en los métodos cuantitativos como en los cualitativos, para así poder concebir interrogantes diferentes que hagan avanzar al campo.

En suma, y estrechamente vinculadas con nuestros objetivos y con los hallazgos reportados en otros estudios previos, nos planteamos las siguientes hipótesis y preguntas de investigación:

- $\mathrm{H}_{1}$ : se producirá un incremento constante en el número de artículos publicados sobre comunicación política durante el periodo 2013-2017.

- $\mathrm{H}_{2}$ : los trabajos más habituales serán de cariz empírico.

- $\mathrm{H}_{3}$ : las teorías más empleadas tendrán que ver con el framing y la agenda-setting.

- $\mathrm{H}_{4}$ : los métodos cuantitativos superarán a los cualitativos, destacando, sobre todos ellos, el análisis de contenido.

- $\mathrm{H}_{5}$ : las unidades de contexto que protagonizarán la investigación académica serán los nuevos medios de comunicación (TIC y redes sociales).

- $\mathrm{PI}_{1}$ : ¿se producirán diferencias según el origen geográfico de las revistas (España frente a Hispanoamérica)?

- $\mathrm{PI}_{2}$ : ¿existirán patrones de publicación característicos de la producción española e hispanoamericana en comunicación política? 


\section{Metodología}

En aras del cumplimiento de los objetivos estipulados y la respuesta a las hipótesis y preguntas formuladas, se puso en marcha una meta-investigación:

Un método descriptivo cuantitativo vinculado a las técnicas del análisis de contenido, especialmente diseñadas para investigar cómo se organiza el "formato" de artículo científico como medio de comunicación y difusión entre públicos especializados (Saperas y Carrasco-Campos, 2019: 222).

Esto es, se planteó un estudio bibliométrico combinado con el análisis de contenido de los artículos académicos divulgados en las principales cabeceras de comunicación del ámbito hispánico. Según Mancinas-Chávez et al. (2016: 255), "los espacios de divulgación de resultados de la investigación en español en el área de comunicación son insuficientes”. Por ello, se acudió al repositorio de SCImago Journal \& Country Rank (2018), donde constan las revistas de mayor prestigio, agrupadas por disciplinas y clasificadas según su factor de impacto. Así pues, las unidades de recogida de datos fueron (ordenadas de mayor a menor impacto): Comunicar, El Profesional de la Información, Communication \& Society, Revista Latina de Comunicación Social, Cuadernos.info, Comunicación y Sociedad y Palabra Clave.

Estas siete revistas son las más destacadas en el lapso de 2013 a 2017, ya que todas ellas figuraban, en el último año considerado, en el primer cuartil de la clasificación o en el segundo. Por su parte, las unidades de análisis representaron una muestra de $n=167$ manuscritos, $\sim 11 \%$ del total de los $N=1548$ artículos publicados en el quinquenio seleccionado en dichas revistas (Piñeiro-Naval y Morais, 2019). Tras una minuciosa revisión a cargo de los autores del estudio, esta muestra fue extractada a partir del universo total por tratarse de trabajos que aludían, precisamente, a la dimensión política de la comunicación. Los parámetros en que se basó dicha extracción tienen que ver (entre otros) con los ejes temáticos y conceptuales repasados en el bloque inicial del presente texto: comunicación política, campañas electorales, uso de medios digitales por parte de actores políticos, estudios sobre marketing, periodismo y política, o investigaciones sobre deliberación cívica, participación ciudadana y engagement.

La plantilla de codificación empleada para el análisis se compuso de las siguientes variables, todas ellas inspiradas en estudios previos de similar naturaleza (Bennett y Pfetsch, 2018; Chung et al., 2013; Gallego, 2017; Graber y Smith, 2005; Hanusch y Vos, 2019; Karpf et al., 2015; Keating y Totzkay, 2019; Lotero-Echeverri et al., 2020; Nielsen, 2014 y 2017; Piñeiro-Naval, 2020; Piñeiro-Naval y Mangana, 2019; Rodríguez-Estrada et al., 2017; Sierra y Rodríguez-Virgili, 2020): 
1. Año en el que se publicó el artículo: desde 2013 hasta 2017.

2. Revista en la que se incluyó el manuscrito.

3. Índice de impacto $S J R$ que presentaba la revista el año en el cual se publicó el artículo.

4. Número de autores que firman el manuscrito.

5. Universidad de pertenencia de los firmantes (en el supuesto de identificarse varias instituciones, se recogió el dato de filiación del primer autor).

6. Tipo de trabajo $\left(\alpha_{k}=0,78\right)$ : empírico, teórico-ensayístico o metodológico.

7. Objeto de estudio $\left(\alpha_{k}=1\right)$ : emisor, mensaje, receptor o estructura del sistema mediático.

8. Teoría o corpus conceptual aludido $\left(\alpha_{k}=0,72\right)$ : para más detalle, véase la Tabla 2.3

9. Metodología empleada $\left(\alpha_{k}=0,73\right)$ : véase la Tabla 2 .

10. Medio o soporte analizado $\left(\alpha_{k}=1\right)$ : véase la Tabla 2 .

11. Muestra de estudio seleccionada $\left(\alpha_{k}=0,81\right)$ : emplea criterios probabilísticos o no.

12. Paradigma epistemológico al que se adhiere el manuscrito $\left(\alpha_{k}=\right.$ 0,91): positivista, cultural, crítico o retórico.

Las cinco primeras variables cumplen una función de caracterización general bibliométrica, mientras que los siete ítems restantes son analíticooperativos. Téngase en cuenta, además, que los datos pertenecientes al índice de impacto de las revistas fueron extraídos, a modo de variables independientes, del portal de SCImago Journal \& Country Rank (2018); una circunstancia que permitió su posterior triangulación (Denzin, 2015) con los datos aquí recabados.

La recogida de estos se llevó a cabo durante noviembre y diciembre de 2018. Para medir la fiabilidad del procedimiento, fue codificada por dos evaluadores una submuestra aleatoria de $n=18$ artículos $(\sim 11 \%$ de la muestra). Para calcular este parámetro indispensable en todo análisis de contenido se empleó el "Alpha de Krippendorff" (Hayes y Krippendorff,

3 Las figuras y las tablas se encuentran en el Anexo, al final del presente artículo (Nota del editor). 
2007; Krippendorff, 2011), que alcanzó un valor promedio muy satisfactorio: $M\left(\alpha_{k}\right)=0,85$ (el $\alpha_{k}$ de cada variable analítico-operativa aparece en el listado anterior).

\section{Resultados}

Comenzando por los parámetros bibliométricos, el número de artículos que han sido divulgados cada año asciende a: $M_{\text {anual }}=33,4$ trabajos $(D T=17,6)$, mientras que su factor de impacto promedio es: $M_{\text {SJR-IF }}=0,432(D T=$ $0,227)$. El Gráfico 1 verifica que tanto el aumento anual de manuscritos $\left(\mathrm{H}_{1}\right)$ $\left[\lambda=0,833 ; F(4 ; 163)=8,17 ; p<0,001 ; \eta^{2}=0,167\right]$ como, de forma muy especial, el incremento de su impacto $\left[F_{\text {SJR-IF xaño }}(4 ; 162)=28,17 ; p<0,001\right.$; $\left.\eta^{2}=0,415\right]$ son muy acusados a lo largo de la serie temporal analizada. De hecho, ambos datos correlacionan de modo muy intenso y significativo $[r$ $(165)=0,633 ; p<0,001]$; por lo tanto, cuanto más se publica, más impacto se genera.

En cuanto a las revistas, la muestra se distribuye de la siguiente manera entre las siete cabeceras que ejercieron como unidades de recogida de datos: Comunicar (1,2\%), El Profesional de la Información (18\%), Communication \& Society (16,8\%), Revista Latina de Comunicación Social (27,5\%), Cuadernos.info (13,2\%), Comunicación y Sociedad (13,8\%) y Palabra Clave (9,6\%). En este sentido, las cabeceras españolas representan $63,5 \%$ de la producción total y las hispanoamericanas se limitan a $36,5 \%$. Si las comparamos respecto al factor de impacto medio durante el lustro analizado, observamos diferencias estadísticamente significativas entre las revistas españolas $\left(M_{\text {SIR-IF }}=0,568\right.$; $D T=0,142)$ e hispanoamericanas $\left(M_{\text {SJR-IF }}=0,195 ; D T=0,135\right)$, y de un tamaño "elevado" $[t(165)=16,63 ; p \stackrel{0}{<} 0,001 ; d=2,69]$ (Cohen, 1988; Johnson et al., 2008).

En lo referente al número de autores que firman cada manuscrito, los valores descriptivos son: $M_{\text {autores }}=2,17(D T=1,01)$. Se observa, en este sentido, que el impacto de los trabajos aumenta a medida que lo hace el número de autores $[r(165)=0,343 ; p<0,001]$. Las instituciones de pertenencia más destacadas son la Universidad de Valladolid, la Universidad Complutense de Madrid y la Universidad de Valencia. De entre los 78 organismos identificados, los 13 que están incluidos en la Tabla 1 son responsables de 46,7\% de la producción total. Por otro lado, todas son españolas salvo la Universidad Autónoma de Nuevo León y la Benemérita Universidad Autónoma de Puebla, ambas mexicanas. 
Desde el punto de vista analítico-operativo, 87,4\% de los trabajos son empíricos, 9\% teórico-ensayísticos y 3,6\% metodológicos $\left(\mathrm{H}_{2}\right)$. Por otro lado, la mayoría de los estudios se enfocan en el mensaje $(74,9 \%)$, en segundo lugar, en la audiencia $(10,8 \%)$, luego en la estructura $(7,8 \%)$ y, en último lugar, en la fuente de la información política (6,6\%). La Tabla 2 condensa los hallazgos relativos a las teorías y métodos empleados, así como a los medios o soportes analizados. En este sentido, el corpus teórico dominante en la investigación centrada en comunicación política es la teoría del framing $(18,6 \%)$ $\left(\mathrm{H}_{3}\right)$. Por otro lado, cabe subrayar que en $29,3 \%$ de los estudios no existe ningún andamiaje conceptual concreto.

En cuanto a los métodos, el análisis de contenido es, sin duda, el predominante $(32,3 \%)$. Si agrupamos los métodos en cuantitativos y cualitativos $\left(\mathrm{H}_{4}\right)$, observamos que $59,3 \%$ de los trabajos se valen de los primeros, mientras que $28,1 \%$ de los segundos (repárese en que 12,6\% de los manuscritos no son de índole empírica). Para terminar con la Tabla 2, y si bien las redes sociales y los demás elementos de la Web 2.0 son los medios protagonistas de la muestra $(33,5 \%)\left(\mathrm{H}_{5}\right)$, los medios tradicionales $(52,7 \%)$ continúan acaparando una mayor atención por parte de los investigadores frente a los nuevos medios $(40,7 \%)$.

Para acabar el repaso porcentual univariable de los ítems analítico-operativos, es preciso aclarar que $65,9 \%$ de los artículos emplean muestras no probabilísticas; sin embargo, $21,6 \%$ sí se basa en reglas estadísticas para el diseño de muestras probabilísticas (recordemos, una vez más, que el restante $12,7 \%$ son trabajos no empíricos). Respecto a los paradigmas epistemológicos, la distribución de los artículos es la siguiente: positivistas (60,5\%), críticos $(18,6 \%)$, culturales $(12,6 \%)$ y retóricos $(8,4 \%)$.

Si procedemos ahora con las comparaciones entre las revistas españolas y las hispanoamericanas $\left(\mathrm{PI}_{1}\right)$ (véase la Tabla 3), y en lo que atañe a la tipología de los trabajos (recordemos: empíricos, teórico-ensayísticos o metodológi$\cos )$, no existen diferencias entre las cabeceras $\left[\chi^{2}(2, N=167)=2,47 ; p=\right.$ $0,291 ; v=0,122]$. Sí se producen en relación con los objetos de estudio $\left[\chi^{2}\right.$ $(3, N=167)=8,84 ; p=0,031 ; v=0,23]$, pues los trabajos publicados en las revistas españolas prestan una mayor atención al mensaje, mientras que las hispanoamericanas aluden al emisor y al receptor. Los paradigmas epistemológicos también exhiben divergencias $\left[\chi^{2}(3, N=167)=9,32 ; p=0,025\right.$; $v=0,236]$, ya que en España se apela más al positivista y en Hispanoamérica al crítico. En esta línea, los métodos cuantitativos son más propios de la primera y los cualitativos de la segunda $\left[\chi^{2}(1, N=146)=7,21 ; p=0,007\right.$; $v=0,222]$. Respecto a los medios, también se observan diferencias $\left[\chi^{2}(1\right.$, 
$N=156)=4,92 ; p=0,026 ; v=0,178]$; en este caso, los etiquetados como "nuevos medios" ostentan un mayor protagonismo en los manuscritos divulgados en revistas españolas, y los tradicionales en las hispanoamericanas.

En otro orden de ideas, y tomando como punto de partida el factor de impacto, resulta de elevado interés contrastar si hay diferencias en función de los métodos empleados en los artículos y los medios protagonistas de estos. A tenor de los datos mostrados en la Figura 2, se observa que los manuscritos donde se emplean métodos cuantitativos $(M=0,472 ; D T=0,209)$ superan en factor de impacto a aquellos con técnicas cualitativas $(M=0,356 ; D T=$ $0,241)$, y lo hacen, además, de manera significativa $[t(144)=2,97 ; p=0,003$; $d=0,514]$ y con un tamaño de efecto "medio" (Cohen, 1988; Johnson et al., 2008). Una situación muy semejante es la que se establece entre los trabajos centrados en el estudio de los medios tradicionales $(M=0,377 ; D T=0,238)$ frente a los nuevos medios $(M=0,517 ; D T=0,189)$, ya que existen claras diferencias entre ambos $[t(154)=-3,98 ; p<0,001 ; d=-0,651]$.

Por último, se puso en marcha una estrategia empleada con anterioridad (Piñeiro-Naval y Morais, 2019), consistente en la ejecución de un análisis de conglomerados bietápico, o Two-Step Cluster Analysis. Este procedimiento, válido tanto para variables continuas como categóricas (Rundle-Thiele et al., 2015), operó con los seis ítems más notables; es decir: la teoría empleada en los manuscritos, ${ }^{4}$ el método, el objeto de investigación, el medio de comunicación en el que se centran, la temática referida y el paradigma al cual se adscriben los estudios de la muestra. La medida de la silueta de cohesión y separación ascendió a "0,3", un valor bastante aceptable (Norušis, 2012). En lo concerniente al peso de los ítems predictores, el método es el elemento más destacado (con valor 1 ), seguido del paradigma epistemológico $(0,58)$, el medio (0,53), la teoría $(0,29)$, el objeto de estudio $(0,27)$ y, en última instancia, el tipo de trabajo $(0,04)$. Los cuatro conglomerados derivados del análisis presentan un coeficiente de tamaño (del más grande al más pequeño) de "1,64", un dato que remite a la homogeneidad de los grupos (Tkaczynski, 2017). Sucintamente, cada uno de estos cuatro patrones de publicación (todos ellos de índole empírica) se caracterizan del siguiente modo (para más detalle, véase la Tabla 4):

4 La variable "teoría” ha sido recodificada para tomar en cuenta únicamente aquellos trabajos que sí han empleado alguna clase de corpus conceptual, considerando al resto como missing. Esta es la razón por la que el total de unidades consideradas para el análisis de conglomerados bietápico asciende a $n=118(70,7 \%$ del total $)$. 
- $\quad 1^{\circ}$ : artículos basados en análisis de contenido que abordan el estudio de los mensajes divulgados en redes sociales, y cuyo sustrato teórico radica en los conceptos propios de la Web 2.0.

- $2^{\circ}$ : estudios mediante análisis crítico del discurso de los encuadres (frames) reflejados en prensa.

- $3^{\circ}$ : trabajos también sobre prensa y framing, aunque, en este caso, investigado con la técnica del análisis de contenido.

- $\quad 4^{\mathrm{o}}$ : manuscritos centrados en los receptores de la comunicación y que, mediante una triangulación metodológica, tratan aspectos relacionados con la agenda-setting de los medios en general.

Estos cuatro conglomerados $\left(\mathrm{PI}_{2}\right.$ ) pueden ser cotejados con arreglo a su factor de impacto. Así, el análisis de la varianza pone de manifiesto la existencia de diferencias entre los grupos $\left[F_{\text {Clusters x SIR-IF }}(3 ; 114)=8,25\right.$; $\left.p<0,001 ; \eta^{2}=0,178\right]$. De manera comparada, y según la prueba post-hoc T3 de Dunnett, los conglomerados $1^{\circ}$ y $2^{\circ}$ son los que manifiestan las mayores diferencias $[t(66)=5,101 ; p<0,001 ; d=1,232]$, caracterizadas por un tamaño elevado atendiendo al effect size (Cohen, 1988; Johnson et al., 2008).

Por fin, fueron cruzados estos cuatro patrones con las revistas donde se publican los artículos. De conformidad con los valores mostrados en la Tabla 5, se observan diferencias significativas $\left[\chi^{2}(3, N=118)=19,42\right.$; $p<0,001 ; v=0,406]$. A tenor de los residuos tipificados corregidos, las revistas hispanoamericanas tienden a incluir, en gran medida, los tipos de artículos $2^{\circ}$ y $4^{\circ}$; mientras que las españolas se decantan más por el caso $1^{\circ}$. En cuanto al $3^{\circ}$, no hay diferencias entre las revistas.

\section{Discusión y conclusiones}

Iniciamos este trabajo con un propósito: examinar la publicación de artículos sobre política en las principales revistas hispánicas de comunicación durante el periodo 2013-2017. Tras recoger los datos y efectuar el análisis, podemos concluir que existe, desde el punto de vista bibliométrico, un notable aumento tanto del número de manuscritos como de su impacto; un hecho que denota la creciente relevancia de este campo de estudio en el marco de las ciencias sociales. Por otra parte, los porcentajes también revelan que son las revistas españolas, en comparación con las hispanoamericanas, las que divulgan una proporción de artículos mayor en relación con la temática que nos ocupa. La inestabilidad política que ha marcado, en los últimos tiempos, la actualidad de los acontecimientos en España con el surgimiento de nuevas formaciones, 
el despertar de movimientos de acción cívica, los múltiples comicios celebrados o las problemáticas acaecidas por causa de la independencia de Cataluña son factores que, posiblemente, justifican los contrastes entre unas cabeceras y otras.

Si reparamos en los resultados relativos a la autoría de los trabajos, podemos determinar que los manuscritos sobre comunicación y política suelen ser colaborativos, ya que presentan más de dos autores de media; un dato que aumenta a medida que lo hace el factor de impacto. Asimismo, parece que los investigadores circunscritos a universidades españolas son más proactivos que sus homólogos hispanoamericanos, donde destacan apenas dos instituciones mexicanas como son la Universidad Autónoma de Nuevo León y la Benemérita Universidad Autónoma de Puebla.

A nivel analítico-operativo, los trabajos empíricos acaparan un mayor protagonismo frente a los teórico-ensayísticos o los de índole metodológica. Esta propensión hacia lo empírico se traduce en la observación de un objeto de estudio recurrente: el mensaje, que relega a los emisores y las audiencias a un segundo plano. Como verificamos en el epígrafe correspondiente al estado del arte, son varios los expertos que reclaman una mayor variedad de aproximaciones para atender a las múltiples aristas de un sistema político muy cambiante. Esta visión crítica podría aplicarse al corpus aquí recopilado, sobre todo si consideramos que los preceptos teóricos con mayor presencia siguen siendo el framing y la agenda-setting, y que la metodología más empleada para el estudio de la información política continúa siendo el análisis de contenido; un recurso que, por otro lado, difícilmente puede abordar el estudio de muestras representativas. Se confirmaría, tal y como advertían algunos autores (Bennett e Iyengar, 2008; Bimber, 2015; Kparf et al., 2015; Nielsen, 2014; Robinson, 2019), una suerte de "consenso paradigmático" en la investigación que trata de las relaciones entre comunicación y política, de modo que el positivista imperaría sobre los restantes paradigmas. Finalmente, se constata que las redes sociales son los medios predilectos de los estudiosos del área, un dato que va en consonancia con las tendencias internacionales que se ocupan de analizar las múltiples conexiones que se establecen entre los new media y los asuntos políticos.

Además de esbozar un perfil general característico de esta producción académica transfronteriza, es muy pertinente que incidamos en las diferencias que existen entre las revistas españolas y las hispanoamericanas. En este sentido, los artículos de las principales cabeceras de España, adscritos al paradigma positivista, suelen incluir trabajos empíricos centrados en el estudio cuantitativo de los mensajes difundidos a través de los nuevos medios. Por el 
contrario, sus homólogas hispanoamericanas presentan, habitualmente, manuscritos que se amparan en el paradigma crítico, y que consisten en estudios focalizados en el análisis cualitativo tanto de los emisores como de los receptores de medios tradicionales.

Desde el punto de vista de los patrones de publicación (denominados aquí "conglomerados" por ser esta su acepción estadística), es posible identificar, para cada origen geográfico, un modelo recurrente de artículo. En España, los autores practican con asiduidad el análisis de contenido (por tanto, un método empírico y positivista) de los mensajes políticos propagados en redes sociales, apoyándose a nivel teórico en los preceptos de la web social o 2.0. En Hispanoamérica, por su parte, los estudiosos en comunicación política se decantan, normalmente, por el análisis crítico de los encuadres (frames) presentes en la información recogida en la prensa tradicional. Estos dos modelos también exhiben grandes diferencias no solamente en lo teórico y metodológico, sino también en lo bibliométrico; esto es, los manuscritos de las revistas españolas alcanzan un factor de impacto más elevado que aquellos publicados en Hispanoamérica.

En otro orden de ideas, la relevancia del presente estudio estriba en el hecho de habernos permitido obtener un mayor conocimiento acerca de esta fecunda área de investigación. Como expresan Alves et al. (2019: 38), "cuando el contexto político se convierte en objeto de transformaciones significativas, nuevos conjuntos de teorías y abordajes metodológicos se hacen necesarios para dar cuenta de la nueva realidad". Sin embargo, hemos observado que la investigación en comunicación política adscrita al ámbito hispánico todavía debe ahondar en la implementación de herramientas innovadoras que permitan explicar nuevos conceptos. Esta adopción debe ser equilibrada para que no sobrevengan ciertos problemas que sí se encuentran en el ámbito anglosajón. Tal y como explican Kosicki et al. (2011: 557-558):

A pesar del tremendo crecimiento en las técnicas metodológicas y los procedimientos de análisis de datos, los investigadores continúan enfrentándose a muchas dificultades potenciales. En su exuberancia por aplicar técnicas y procedimientos vanguardistas de análisis, algunos investigadores muestran una tendencia a esconderse detrás del método con el fin de elevar la sofisticación metodológica sobre la sustancia teórica.

Por tanto, la progresiva refinación de los métodos no debería acarrear un hermetismo que juegue en detrimento del propósito último para el cual fueron ideados: resolver problemas teóricos que permitan el avance de la disciplina.

En cuanto a las limitaciones del estudio, que convendría subsanar de cara a futuros trabajos, es necesario reconocer varias cuestiones. La primera 
tiene que ver con la muestra, pues es altamente probable que parte de la producción generada por los autores afincados en España e Hispanoamérica se haya divulgado en cabeceras ajenas a estos contextos geográficos. Del mismo modo, otras revistas englobadas en dominios como la ciencia política o las artes y humanidades también acostumbran ser vehículos de difusión de la investigación en comunicación política. Por otro lado, y en aras de obtener una radiografía más precisa del impacto de los artículos, sería muy aconsejable tomar el dato de las citas directas recibidas para cada uno de los manuscritos, lo cual permitiría refinar tanto los análisis tendenciales como comparativos. Teniendo en cuenta estos aspectos, las sucesivas meta-investigaciones que llevemos a cabo gozarán de una capacidad descriptiva y explicativa mayor.

\section{Referencias}

Alves, Marcelo et al. (2019), "Datificação e redes na comunicação política: mapeamento de redes e fluxos no Facebook", en Revista Esferas, núm. 14, Brasil: Universidades do Centro Oeste.

Ballesteros, Carlos A. y Díez-Garrido, Marta (2018), "We Need to Talk. Engagement 2.0 on Facebook during the Spanish Cyber Campaign of December 20, 2015", en Communication \& Society, vol. 31, núm. 1, España: Universidad de Navarra.

Bennett, Lance W. e Iyengar, Shanto (2008), "A new era of minimal effects? The changing foundations of political communication", en Journal of Communication, vol. 58, núm. 4, Reino Unido: Oxford University Press.

Bennett, Lance W. y Pfetsch, Barbara (2018), "Rethinking Political Communication in a Time of Disrupted Public Spheres", en Journal of Communication, vol. 68, núm. 2, Reino Unido: Oxford University Press.

Bimber, Bruce (2015), "What's next? Three challenges for the future of political communication research", en Gil-de-Zúñiga, Homero [ed.], New technologies and civic engagement. New Agendas in Communication, Reino Unido: Routledge.

Bucy, Erik P. y Holbert, Lance (2013), Sourcebook for Political Communication Research. Methods, Measures, and Analytical Techniques, Estados Unidos: Routledge.

Campos-Domínguez, Eva (2017), “Twitter y la comunicación política”, en El Profesional de la Información, vol. 26, núm. 5, España: El Profesional de la Información.

Chung, Chung Joo et al. (2013), "An analysis on communication theory and discipline", en Scientometrics, núm. 95, Suiza: Springer.

Cohen, Jacob (1988), Statistical power analysis for the behavioral sciences, Estados Unidos: Lawrence Erlbaum.

Correia, João Carlos et al. (2010), Conceitos de Comunicação Política, Portugal: Livros LabCom.

Denzin, Norman (2012), "Triangulation 2.0", en Journal of Mixed Methods Research, vol. 6, núm. 2, Estados Unidos: Sage.

Denzin, Norman (2015), “Triangulation”, en Ritzer, George [ed.], The Blackwell Encyclopedia of Sociology, Estados Unidos: John Wiley \& Sons. 
Gallego, José Raúl (2017), "Existe... pero no se ve. Investigación de Agenda Setting en América Latina”, en La Trama de la Comunicación, vol. 21, núm. 2, Argentina: Universidad Nacional del Rosario.

Graber, Doris y Smith, James (2005), "Political Communication faces the 21st century", en Journal of Communication, vol. 55, núm. 3, Reino Unido: Oxford University Press.

Hanusch, Folker y Vos, Tim (2019), "Charting the development of a field: A systematic review of comparative studies of journalism", en The International Communication Gazette, vol. 82, Estados Unidos: Sage.

Hayes, Andrew y Krippendorff, Klaus (2007), "Answering the call for a standard reliability measure for coding data", en Communication Methods and Measures, vol. 1, núm. 1, Reino Unido: Taylor \& Francis.

Jamieson, Kathleen y Kenski, Kate (2017), "Political Communication: Then, Now, and Beyond", en Kenski, Kate y Jamieson, Kathleen [eds.], The Oxford Handbook of Political Communication, Estados Unidos: Oxford University Press.

Johnson, Blair et al. (2008), "Contemporary approaches to meta-analysis in communication research”, en Hayes, Andrew et al. [eds.], The Sage Sourcebook of Advanced Data Analysis Methods for Communication Research, Estados Unidos: Sage.

Karpf, Dave et al. (2015), "The Role of Qualitative Methods in Political Communication Research: Past, Present, and Future", en International Journal of Communication, núm. 9, Estados Unidos: USC Annenberg.

Keating, David y Totzkay, Daniel (2019), "We do publish (conceptual) replications (sometimes): publication trends in communication science, 2007-2016", en Annals of the International Communication Association, Estados Unidos: Taylor \& Francis.

Kosicki, Gerald et al. (2011), "Looking Back and Looking Forward: Observations on the Role of Research Methods in the Rapidly Evolving Field of Political Communication", en Bucy, Erik y Holbert, Lance [eds.], The Sourcebook for Political Communication Research Methods, Measures, and Analytical Techniques, Estados Unidos e Inglaterra: Routledge.

Krippendorff, Klaus (2011), "Agreement and information in the reliability of coding", en Communication Methods and Measures, vol. 5, núm. 2, Reino Unido: Taylor \& Francis.

Lasswell, Harold (1927), Propaganda Technique in the World War, Estados Unidos: Peter Smith.

Lazarsfeld, Paul et al. (1968), The people's choice: How the voter makes up his mind in an election campaign, Estados Unidos: Columbia University Press.

Lippmann, Walter (1922), Public Opinion, Estados Unidos: Harcourt, Brace \& Company.

Lippmann, Walter (1925), The Phantom Public, Estados Unidos: Harcourt, Brace \& Company.

Lotero-Echeverri, Gabriel et al. (2020), "Análisis de investigaciones iberoamericanas en el campo de la comunicación y la opinión pública”, en Correspondencias \& Análisis, núm. 11, Perú: Universidad San Martín de Porres.

Mancinas-Chávez, Rosalba et al. (2016), "Problemas de la divulgación de las investigaciones en Comunicación en revistas de alto impacto en español”, en Revista F@ro, vol. 1, núm. 23, Chile: Universidad de Playa Ancha.

Muñiz, Carlos et al. (2016), “¿Están los políticos políticamente comprometidos?: Análisis del compromiso político 2.0 desarrollado por los candidatos a través de Facebook”, en Cuadernos.info, núm. 39, Chile: Pontificia Universidad Católica. 
Nielsen, Rasmus (2014), "Political communication research: New media, new challenges, and new opportunities", en MedieKultur: Journal of Media and Communication Research, vol. 30, núm. 56, Dinamarca: Society of Media researchers In Denmark.

Nielsen, Rasmus (2017), "No One Cares What We Know: Three Responses to the Irrelevance of Political Communication Research", en Political Communication, vol. 35, núm. 1, Estados Unidos: Taylor \& Francis.

Nimmo, Dan y Sanders, Keith [eds.] (1981), Handbook of political communication, Estados Unidos: Sage.

Norušis, Marija (2012), IBM SPSS statistics 19 guide to data analysis, Estados Unidos: Prentice Hall.

Pérez-Montoro, Mario (2016), “Gestión del conocimiento: orígenes y evolución”, en El Profesional de la Información, vol. 25, núm. 4, España: El Profesional de la Información.

Piñeiro-Naval, Valeriano (2020), "The content analysis methodology. Uses and applications in communication research on Spanish-speaking countries", en Communication \& Society, vol. 33, núm. 3, España: Universidad de Navarra.

Piñeiro-Naval, Valeriano y Mangana, Rafael (2019), "La presencia del framing en los artículos publicados en revistas hispanoamericanas de comunicación indexadas en Scopus”, en Palabra Clave, vol. 22, núm. 1, Colombia: Universidad de La Sabana.

Piñeiro-Naval, Valeriano y Morais, Ricardo (2019), "Estudio de la producción académica sobre comunicación en España e Hispanoamérica”, en Comunicar, vol. 27, núm. 61, España: Grupo Comunicar.

Prior, Hélder et al. (2016), "Comunicação e Política no espaço público lusófono”, en Prior, Hélder et al. [orgs.], Diálogos Lusófonos em Comunicação e Politica, Portugal: Livros LabCom.

Restrepo-Echavarría, Néstor Julián (2019), "La comunicación política: un estudio multidisciplinar desde las ciencias sociales”, en Correspondencias \& Análisis, núm. 10, Perú: Universidad San Martín de Porres.

Robinson, Piers Gregory (2019), "Expanding the Field of Political Communication: Making the Case for a Fresh Perspective Through Propaganda Studies", en Frontiers in Communication, vol. 4, núm. 26, Suiza: Frontiers.

Rodríguez-Andrés, Roberto (2016), "Retos de la comunicación política 2.0 en una sociedad que envejece”, en Revista Mediterránea de Comunicación, vol. 7, núm. 1, España: Universidad de Alicante.

Rodríguez-Estrada, Alejandra et al. (2017), "La tensión centro-periferia en la producción del campo de la comunicación política. El caso mexicano", en Revista Latina de Comunicación Social, núm. 72, España: Sociedad Latina de Comunicación.

Rogers, Everett (2004), “Theoretical Diversity in Political Communication”, en Kaid, Lynda Lee [ed.], Handbook of Political Communication Research, Estados Unidos: Lawrence Erlbaum Associates.

Rundle-Thiele, Sharyn et al. (2015), "Using two-step cluster analysis to identify homogeneous physical activity groups", en Marketing Intelligence \& Planning, vol. 33, núm. 4, Reino Unido: Emerald.

Saperas, Enric y Carrasco-Campos, Ángel (2019), “¿Cómo investigamos la comunicación? La meta-investigación como método para el estudio de las prácticas de investigación en los artículos publicados en revistas científicas", en Sierra Caballero, Francisco y Alberich Pascual, Jordi [eds.], Epistemología de la comunicación y cultura digital: retos emergentes, España: Universidad de Granada. 
SCImago Journal \& Country Rank (2018), Journal Rankings. Disponible en: http://bit. ly/2rc6ELb [10 de octubre de 2018].

Sierra, Aurken y Rodríguez-Virgili, Jordi (2020), "Comunicación política y medios sociales: análisis del estado de la cuestión de 2008 a 2018”, en Revista Mediterránea de Comunicación, vol. 11, núm. 1, España: Universidad de Alicante.

Tkaczynski, Aaron (2017), "Segmentation Using Two-Step Cluster Analysis", en Dietrich, Timo et al. [eds.], Segmentation in Social Marketing, Singapur: Springer.

\section{Anexo}

\section{Figura 1}

\section{Evolución anual promedio de los artículos y su factor de impacto SJR}

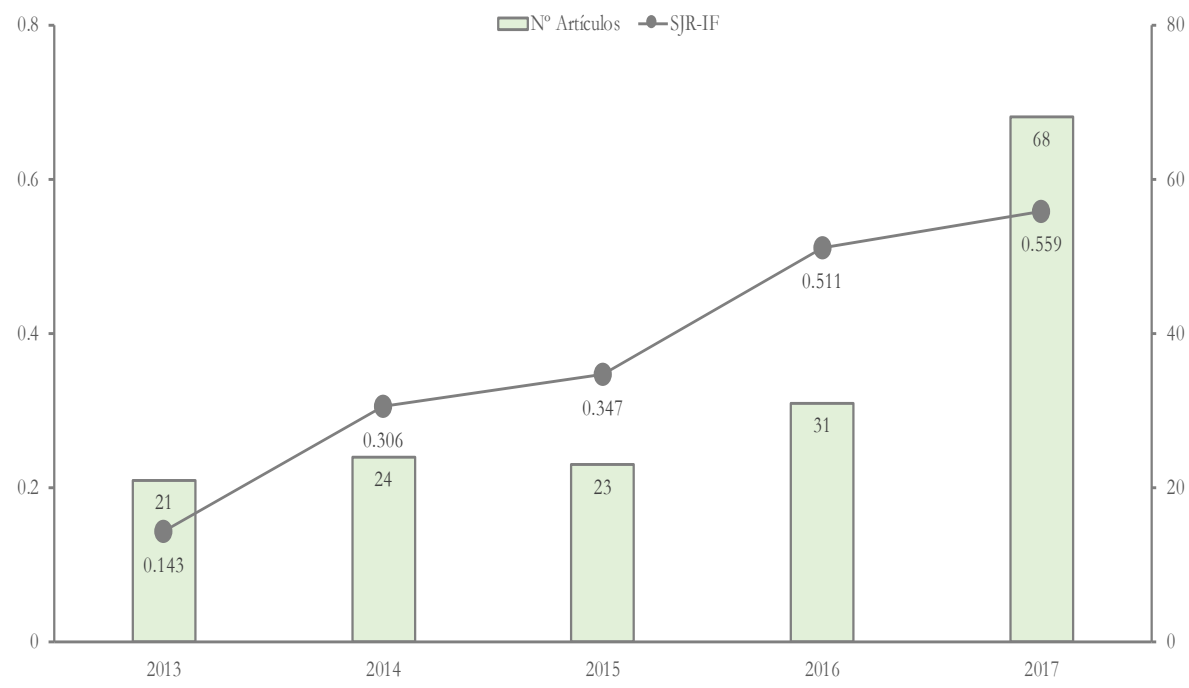

Fuente: Elaboración propia a partir de los datos primarios del estudio y los índices de impacto (datos secundarios) tomados de SCImago Journal \& Country Rank (2018). 


\section{Tabla 1}

Universidades más destacadas de filiación de los autores

\begin{tabular}{lc}
\hline Universidad & $\begin{array}{c}\text { Núm. } \\
\text { artículos }\end{array}$ \\
\hline Universidad de Valladolid & 12 \\
\hline Universidad Complutense de Madrid & 9 \\
\hline Universidad de Valencia & 9 \\
\hline Universidad de Málaga & 6 \\
\hline Universidad de Santiago de Compostela & 6 \\
\hline Universidad Rey Juan Carlos & 6 \\
\hline Universidad Autónoma de Nuevo León & 5 \\
\hline Universidad del País Vasco & 5 \\
\hline Benemérita Universidad Autónoma de Puebla & 4 \\
\hline Universidad Autónoma de Barcelona & 4 \\
\hline Universidad Carlos III de Madrid & 4 \\
\hline Universidad de Vigo & 4 \\
\hline Universidad Pompeu Fabra & 4 \\
\hline Total & 78 \\
\hline
\end{tabular}

Fuente: Elaboración propia a partir de los datos primarios del estudio. 


\section{Tabla 2}

Teorías o conceptos, métodos y medios o soportes

\begin{tabular}{|c|c|c|c|c|c|}
\hline Teorías/conceptos & $\%$ & Métodos & $\%$ & Medios/soportes & $\%$ \\
\hline Framing & 18,6 & $\begin{array}{l}\text { Análisis de } \\
\text { contenido }\end{array}$ & 32,3 & $\begin{array}{l}\text { Redes sociales, Web } \\
2.0\end{array}$ & 33,5 \\
\hline Agenda-setting & 12 & $\begin{array}{l}\text { Análisis del } \\
\text { discurso }\end{array}$ & 13,8 & Prensa & 23,4 \\
\hline Web 2.0 & 10,2 & Estudio de caso & 11,4 & Medios en general & 14,3 \\
\hline Engagement & 7,8 & Triangulación & 8,3 & Televisión & 9,6 \\
\hline Info/Politainment & 4,8 & Encuesta & 7,2 & Internet, TIC & 6,6 \\
\hline $\begin{array}{l}\text { Teorías narrativo- } \\
\text { cinematográficas }\end{array}$ & 3,6 & $\begin{array}{l}\text { Análisis de } \\
\text { contenido } \\
\text { automatizado }\end{array}$ & 4,8 & $\begin{array}{l}\text { Anuncios } \\
\text { publicitarios }\end{array}$ & 3,6 \\
\hline $\begin{array}{l}\text { Exposición } \\
\text { selectiva }\end{array}$ & 2,4 & $\begin{array}{l}\text { Análisis } \\
\text { bibliométrico }\end{array}$ & 3 & Cine & 3 \\
\hline $\begin{array}{l}\text { Teorías identidad } \\
\text { social }\end{array}$ & 1,8 & Análisis de redes & 3 & Receptores & 2,4 \\
\hline Transmedia & 1,8 & Entrevistas & 1,8 & Revistas & 1,8 \\
\hline Estudios culturales & 1,2 & $\begin{array}{l}\text { Estudio } \\
\text { etnográfico }\end{array}$ & 1,2 & Artículos científicos & 0,6 \\
\hline Otros 5 & 6,5 & $\begin{array}{l}\text { Análisis } \\
\text { heurísticos }\end{array}$ & 0,6 & Radio & 0,6 \\
\hline $\begin{array}{l}\text { No se usa marco } \\
\text { teórico concreto }\end{array}$ & 29,3 & $\begin{array}{l}\text { No es un trabajo } \\
\text { empírico }\end{array}$ & 12,6 & Videojuegos & 0,6 \\
\hline Total & 100 & Total & 100 & Total & 100 \\
\hline
\end{tabular}

Fuente: Elaboración propia a partir de los datos primarios del estudio.

5 Otras teorías y conceptos detectados han sido: teoría de los usos y gratificaciones, modelos de persuasión narrativa, alfabetización mediática, preceptos del diseño web (arquitectura de la información, usabilidad, accesibilidad, etc.), responsabilidad social corporativa y branding. 


\section{Tabla 3}

Comparaciones múltiples en función del origen de las revistas

\begin{tabular}{|c|c|c|c|}
\hline \multirow{2}{*}{ Parámetros de análisis } & \multirow{2}{*}{$\%$ Total } & \multicolumn{2}{|c|}{ Origen de las revistas } \\
\hline & & España & Hispanoamérica \\
\hline \multicolumn{4}{|c|}{ - Tipología de los trabajos: } \\
\hline Empíricos & 87,4 & 88,7 & 85,2 \\
\hline Teórico-ensayísticos & 9 & 9,4 & 8,2 \\
\hline Metodológicos & 3,6 & 1,9 & 6,6 \\
\hline \multicolumn{4}{|l|}{ - Objetos de estudio: } \\
\hline Emisor & 6,6 & $3,8-$ & $11,5+$ \\
\hline Mensaje & 74,9 & $82,1+$ & $62,3-$ \\
\hline Receptor & 10,8 & $7,5-$ & $16,4+$ \\
\hline Estructura & 7,8 & 6,6 & 9,8 \\
\hline \multicolumn{4}{|c|}{ - Paradigmas epistemológicos: } \\
\hline Positivista & 60,5 & $68,9+$ & $45,9-$ \\
\hline Cultural & 12,6 & 11,3 & 14,8 \\
\hline Crítico & 18,6 & $13,2-$ & $27,9+$ \\
\hline Retórico & 8,4 & 6,6 & 11,5 \\
\hline $\mathrm{N}$ & 167 & 106 & 61 \\
\hline \multicolumn{4}{|c|}{ - Métodos de investigación: } \\
\hline Cuantitativos & 67,8 & $75,5+$ & $53,8-$ \\
\hline Cualitativos & 32,2 & $24,5-$ & $46,2+$ \\
\hline $\mathrm{N}$ & 146 & 94 & 52 \\
\hline \multicolumn{4}{|l|}{ - Medios protagonistas: } \\
\hline Tradicionales & 56,4 & $50-$ & $68,5+$ \\
\hline Nuevos & 43,6 & $50+$ & $31,5-$ \\
\hline $\mathrm{N}$ & 156 & 102 & 54 \\
\hline
\end{tabular}

Fuente: Elaboración propia a partir de los datos primarios del estudio. 


\section{Figura 2}

\section{Comparaciones según el factor de impacto SJR para los tipos de métodos y medios}

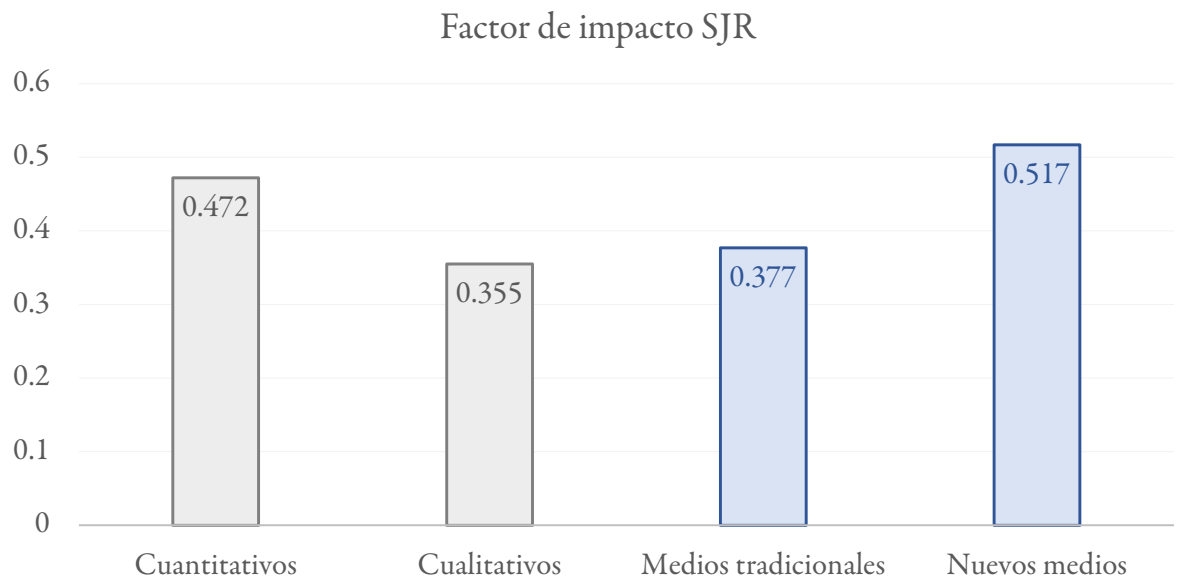

Fuente: Elaboración propia a partir de los datos primarios del estudio y los índices de impacto (datos secundarios) tomados de SCImago Journal \& Country Rank (2018). 


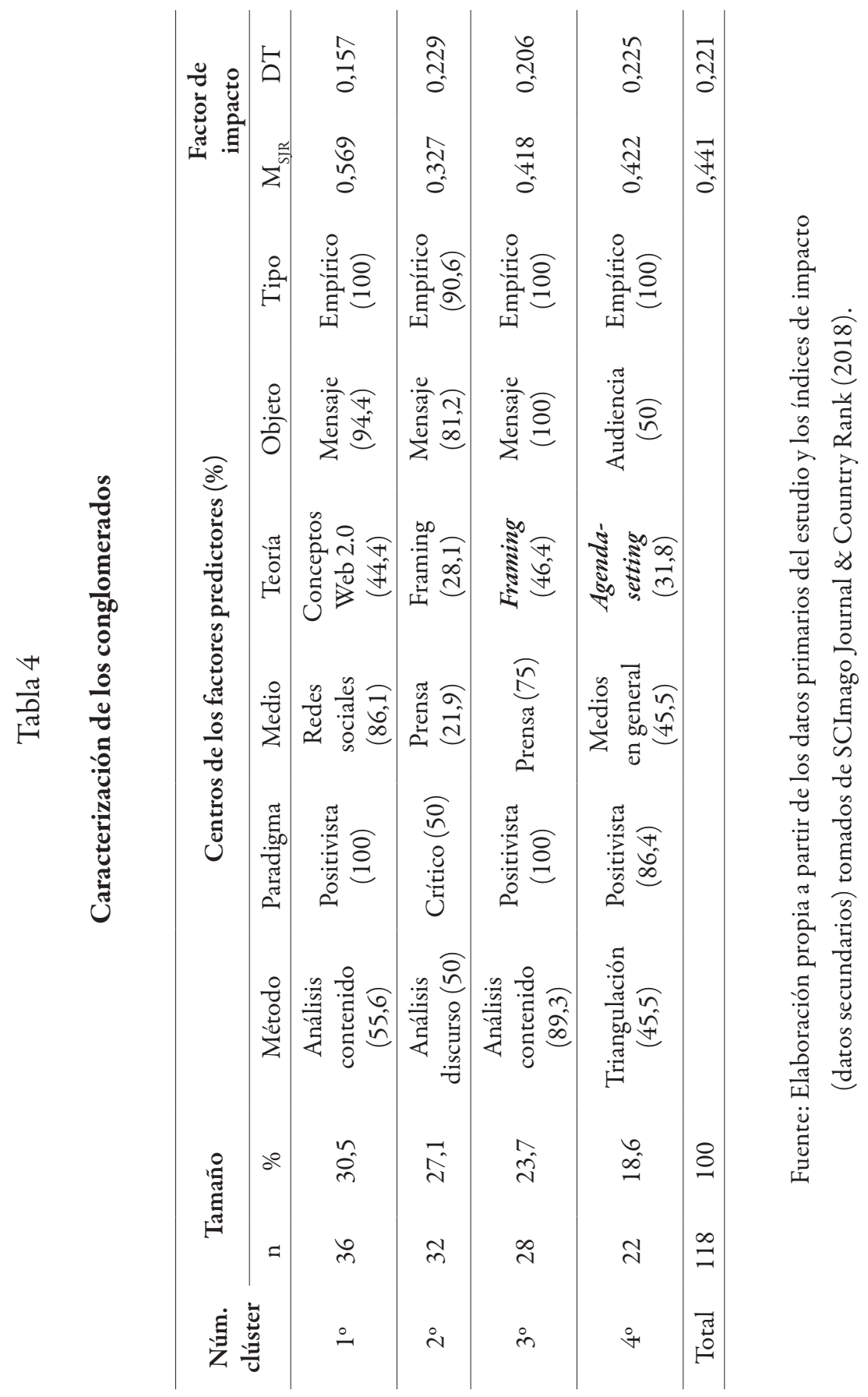




\section{Tabla 5}

\section{Conglomerados según las revistas (\% columna)}

\begin{tabular}{cccc}
\hline \multirow{2}{*}{ Núm. conglomerado } & \% Total & \multicolumn{2}{c}{ Origen de las revistas } \\
\cline { 3 - 4 } & & España & Hispanoamérica \\
\hline${\text { Conglomerado } 1^{\circ}}^{\text {Conglomerado 2 }}$ \% $^{\circ}$ & 30,5 & $42,1+$ & $9,5-$ \\
Conglomerado 3 $^{\circ}$ & 27,1 & $18,4-$ & $42,9+$ \\
Conglomerado 4 & 23,7 & 26,3 & 19 \\
\hline $\mathrm{N}$ & 18,6 & $13,2-$ & $28,6+$ \\
\hline - Valor estadísticamente menor (análisis de los residuos tipificados corregidos). \\
+ Valor estadísticamente mayor (análisis de los residuos tipificados corregidos).
\end{tabular}

Fuente: Elaboración propia a partir de los datos primarios del estudio. 
Valeriano Piñeiro-Naval. Doctor Europeus en Comunicación por la Universidad de Salamanca, España. Actualmente forma parte de la unidad de I\&D LabCom-Communication \& Arts de la Universidade da Beira Interior (Covilhã), donde disfruta de una beca nacional de posdoctorado de la Fundação para a Ciência e a Tecnologia (Ref./número: SFRH/ BPD/122402/2016) de Portugal. Líneas de investigación: patrimonio cultural, turismo y TIC, y meta-investigación en comunicación. Publicaciones recientes: Piñeiro-Naval, Valeriano y Morais, Ricardo (2019), "Estudio de la producción académica sobre comunicación en España e Hispanoamérica”, en Comunicar, vol. 27, núm. 61, España: Grupo Comunicar; Piñeiro-Naval, Valeriano y Serra, Paulo (2019), "How do destinations frame cultural heritage? Content analysis of Portugal's municipal websites", en Sustainability, vol. 11, núm. 4, Suiza: Multidisciplinary Digital Publishing Institute; PiñeiroNaval, Valeriano (2020), "The content analysis methodology. Uses and applications in communication research on Spanish-speaking countries”, en Communication \& Society, vol. 33, núm. 3, España: Universidad de Navarra.

Ricardo Morais. Doctor en Ciencias de la Comunicación y Máster en Periodismo por la Universidade da Beira Interior (Covilhã, Portugal), donde ejerce como profesor asistente en la Facultad de Artes y Letras impartiendo las asignaturas de Periodismo radiofónico y televisivo. También es miembro integrado de la unidad de I\&D LabCom-Communication \& Arts. Líneas de investigación: periodismo, nuevos medios y participación, y comunicación política. Publicaciones recientes: Piñeiro-Naval, Valeriano y Morais, Ricardo (2019), "Estudio de la producción académica sobre comunicación en España e Hispanoamérica”, en Comunicar, vol. 27, núm. 61, España: Grupo Comunicar; Sousa, João, Reis, Bruno y Morais, Ricardo (2019), "Facebook e mobilização cívica em tempos de Troika”, en Lopes, Paula y Reis, Bruno [eds.], Comunicação digital: media, práticas e consumos, Portugal: Núcleo de Investigação em Práticas e Competências Mediáticas \& Universidade Autónoma de Lisboa; Correia, João Carlos, Gradim, Anabela y Morais, Ricardo (2020), Pathologies and dysfunctions of democracy in the media context, Portugal: LabCom Books. 